\title{
FIXED POINT FREE ACTIONS OF SPHERES AND EQUIVARIANT MAPS
}

\author{
ANJU KUMARI AND HEMANT KUMAR SINGH
}

\begin{abstract}
This paper generalizes the concept of index and co-index and some related results for free actions of $G=\mathbb{S}^{0}$ on a paracompact Hausdorff space which were introduced by Conner and Floyd 1 . We define the index and co-index of a finitistic free $G$-space $X$, where $G=\mathbb{S}^{d}, d=1$ or 3 and prove that the index of $X$ is not more than the mod 2 cohomology index of $X$. We observe that the index and co-index of a $(2 n+1)$ sphere $\mathbb{S}^{2 n+1}$ (resp. $(4 n+3)$-sphere $\left.\mathbb{S}^{4 n+3}\right)$ for the action of componentwise multiplication of $G=\mathbb{S}^{1}$ (resp. $\mathbb{S}^{3}$ ) is $n$.

We also determine the orbit spaces of free actions of $G=\mathbb{S}^{3}$ on a finitistic space $X$ with the mod 2 cohomology and the rational cohomology product of spheres $\mathbb{S}^{n} \times \mathbb{S}^{m}, 1 \leq n \leq$ $m$. The orbit spaces of circle actions on the mod 2 cohomology $X$ is also discussed. Using these calculation, we obtain an upper bound of the index of $X$ and the Borsuk-Ulam type results.
\end{abstract}

\section{INTRODUCTION}

Firstly, in 1954, C. T. Yang [27] defines an index for compact Hausdorff spaces with free involutions using Smith (co)homology to study mappings from spheres to euclidean spaces like Borsuk-Ulam theorem and extension of Dyson's theorem. In 1960, Conner and Floyd[1] defines $\mathbb{Z}_{2}$-index (which is Yang's B-index[28]), co-index and homology index, and also discussed the stability of index for Hausdorff spaces. In 1962 2, they discussed the coindex of space of paths $P\left(\mathbb{S}^{n}\right)$ except for some values of $n$. In 1972, Jack Ucci[26] discussed the co-index for remaining values. In 1988, Fadell and Husseni [6] introduced the ideal valued index for free Lie group actions on paracompact spaces. After this many author generalized index in different ways. In 1989, Stolz[21] studied the Conner and Floyd's index for real projective spaces. Volvikov 29](2000) defined numerical index $i_{G}(X)$ using spectral sequences and ideal valued index with filtration for a free $G$-space using the Borel construction for compact Lie group $G$. Jaworowski[10] (2002) defines $G$-index $\left(G=\mathbb{S}^{1}\right.$ or $\mathbb{S}^{3}$ ) with integer coefficients similar to Conner and Floyd's[1] homology index and proved that the index of $(2 n+1)$-lens space is $n$. We call Jaworowski's $G$-index with $\mathbb{Z}_{2}$ coefficient as the mod 2 cohomology $G$-index of $X[20,11]$. Tanaka [23, 24] defined index and co-index for vector bundles and its properties. In 2005, Yasuhiro Hara[7] studies equivariant maps between Stiefel manifolds using index. In 2013, Satya Deo[3] proved that the numerical

2010 Mathematics Subject Classification. Primary 55R25; Secondary 55R10, 55N05 .

Key words and phrases. Free action; Finitistic space; Gysin sequence; Characteristic classes; Classifying maps.

This paper is supported by the Science and Engineering Research Board (Department of Science and Technology, Government of India) with reference number- EMR/2017/002192. 
index for a finitistic space with $p$-torus actions is finite. In 2014, Benjamin Matschke[13] defined ideal valued index using spectral sequences. We generalize Conner and Floyd's 1 ] index and co-index and related standard results to index and co-index for finitistic space $X$ with free actions of $G=\mathbb{S}^{d}, d=1$ or 3 . We also prove that index of $X$ is not mare than the mod 2 cohomology index of $X$.

H. Hopf in 1925-26, raised the question to classify all manifolds whose universal cover is $\mathbb{S}^{n}$. It is equivalent to determine the orbit spaces $\mathbb{S}^{n} / G$, where $G$ is a finite cyclic group. The orbit spaces of $n$-sphere $\mathbb{S}^{n}$ with finite group actions have been studied in [12, 16, 17, 19]. In 1963, J. C. Su[22] computed the orbit spaces of spheres for free circle actions. For the product of spheres $\mathbb{S}^{n} \times \mathbb{S}^{m}$, the orbit spaces of $G=\mathbb{Z}_{p}, p$ a prime with $\mathbb{Z}_{p}$-coefficients or $\mathbb{S}^{1}$-actions with rational coefficients are discussed in [5, 18, 25]. In 1972, Ozeki and Uchida[15] determine the orbit space of free circle action on a manifold with integral cohomology $\mathbb{S}^{2 n+1} \times \mathbb{S}^{2 m+1}$. J Kaur et.al[11] (2015) studied the fixed point free $\mathbb{S}^{3}$ actions on spheres. We generalize the discussion for free actions of $G=\mathbb{S}^{3}$ on $n$-sphere $\mathbb{S}^{n}$ to a finitistic space $X$ with the mod 2 cohomology and the rational cohomology isomorphic to the product of spheres $\mathbb{S}^{n} \times \mathbb{S}^{m}, 1 \leq n \leq m$, and determined the cohomological structure of the orbit spaces. The orbit spaces of $X$ with free actions of $G=\mathbb{S}^{1}$ with $\mathbb{Z}_{2}$ coefficients is also discussed. Using these calculations, we obtain an upper bound of the index of $X$ and Borsuk-Ulam type results.

\section{Preliminaries}

Let $G$ be a compact Lie group. Then by using Milnor's construction[9], there exist a universal principal $G$-bundle $G \hookrightarrow E_{G} \rightarrow B_{G}$. If $X$ is a free $G$-space then the associated bundle $X \stackrel{i}{\hookrightarrow} X_{G}=\frac{X \times E_{G}}{G} \stackrel{\pi}{\longrightarrow} B_{G}$ is a fibre bundle with fibre $G$ where $G$ acts on $X \times E_{G}$ by the diagonal action. This associated fibre bundle becomes a fibration as $B_{G}$ is a paracompact space and this fibration is called the Borel fibration. Then, there exist Leray-Serre spectral sequence associated to this Borel fibration $X \hookrightarrow X_{G} \rightarrow B_{G}$ which has $E_{2}^{k, l}=H^{k}\left(B_{G} ; \mathcal{H}^{l}(X)\right)$, the cohomology of the base $B_{G}$ with local coefficients in the cohomology of the fibre of map $\pi$. Note that if $\pi_{1}\left(B_{G}\right)$ acts trivially on $X$ then $E_{2}^{k, l}=H^{k}\left(B_{G}\right) \otimes H^{l}(X)$.

Proposition 2.1. [14, Theorem 5.9] Let $X \stackrel{i}{\hookrightarrow} X_{G} \stackrel{\pi}{\longrightarrow} B_{G}$ be the Borel fibration. Suppose that the system of local coefficients on $B_{G}$ is simple, then the edge homomorphisms are the homomorphisms $\pi^{*}: H^{k}\left(B_{G}\right) \rightarrow H^{k}\left(X_{G}\right)$ and $i^{*}: H^{l}\left(X_{G}\right) \rightarrow H^{l}(X)$.

For details about the results related to the spectral sequence, we refer [14].

Let $G$ be a compact Lie group which act freely on a finitistic space $X$ and $h: X_{G} \rightarrow$ $X / G$ be the map induced by the $G$-equivariant projection $X \times E_{G} \rightarrow X$. Then $h$ induces an isomorphism on cohomology. Further, $X / G$ and $X_{G}$ have same homotopy type [4]. 
In this paper, we have taken all actions as left actions, all spaces $X$ are assumed to be finitistic space and $H^{*}(X ; R)$ is notation for the Čech cohomology with coefficients in $R$, where $R=\mathbb{Z}_{2}$ or $\mathbb{Q}$. Note that $X \sim_{R} Y$ means $H^{*}(X ; R) \cong H^{*}(Y ; R)$.

The following results has also been used in this paper.

Proposition 2.2. ([20, 11]) Let $X$ be a finitistic space with free $G=\mathbb{S}^{1}$ or $\mathbb{S}^{3}$ action. If $H^{i}(X ; R)=0$ for all $i>n$ then $H^{i}(X / G ; R)=0$ for all $i>n$.

Proposition 2.3. ([8]) Let $R$ denotes a ring and $\mathbb{S}^{n-1} \rightarrow E \stackrel{p}{\rightarrow} B$ be an oriented sphere bundle. Then the following sequence is exact with coefficients in $R$

$$
\cdots \rightarrow H^{i}(E) \stackrel{\rho}{\rightarrow} H^{i-n+1}(B) \stackrel{\cup}{\rightarrow} H^{i+1}(B) \stackrel{p^{*}}{\rightarrow} H^{i+1}(E) \stackrel{\rho}{\rightarrow} H^{i-n+2}(B) \rightarrow \cdots
$$

which start with

$$
0 \rightarrow H^{n-1}(B) \stackrel{p *}{\rightarrow} H^{n-1}(E) \stackrel{\rho}{\rightarrow} H^{0}(B) \stackrel{\cup}{\rightarrow} H^{n}(B) \stackrel{p^{*}}{\rightarrow} H^{n}(E) \rightarrow \cdots
$$

where $\cup: H^{i}(B) \rightarrow H^{i+n}(B)$ maps $x \rightarrow x \cup u$ and $u \in H^{n}(B)$ denotes the characteristic class of the sphere bundle. This sequence is called the Gysin sequence. Observe that $p^{*}: H^{i}(E) \rightarrow H^{i}(B)$ is an isomorphism for all $0 \leq i<n-1$.

\section{INDEX AND CO-INDEX OF $\mathbb{S}^{1}$ AND $\mathbb{S}^{3}$-SPACES}

Recall that for $G=\mathbb{S}^{d}, d=1$ or 3 , the universal principal $\mathbb{S}^{d}$-bundle is $\mathbb{S}^{d} \hookrightarrow \mathbb{S}^{\infty} \rightarrow$ $\mathbb{F P} \mathbb{P}^{\infty}$, where $\mathbb{F}=\mathbb{C}$ or $\mathbb{H}$. Let $G=\mathbb{S}^{d}, d=1$ or 3 acts freely on $\mathbb{S}^{(d+1) k+d}$ by the standard action namely the component wise multiplication. Note that for a topological group $G$ and $G$-space $X$ there exist a $G$-equivariant map $f: G \rightarrow X$ defined as $f(g)=g \cdot x_{0}$ where $x_{0}$ is any fixed element of $X$ and $G$ acts on itself by the group multiplication. In particular, for a $G$-space $X$ there exist an $G$-equivariant map $f: G \rightarrow X$.

Now, we define index and co-index of a free $G$-space $X$. We denote it by $\operatorname{ind}_{G} X$ and co-ind ${ }_{G} X$, respectively.

Definition 3.1. Let $X$ be a free $G$-space, where $G=\mathbb{S}^{d}, d=1$ or 3 . We define index of $X$ as

$$
\operatorname{ind}_{G} X=\max \left\{k \mid \text { there exists an } G \text {-equivariant map } f: \mathbb{S}^{(d+1) k+d} \rightarrow X, k \geq 0\right\} .
$$

It is easy to see that $\operatorname{ind}_{G} X \geq 0$. If there is no upper bound for equivariant maps from spheres to $X$ then $\operatorname{ind}_{G} X=+\infty$.

Definition 3.2. Let $X$ be a free $G$-space, where $G=\mathbb{S}^{d}, d=1$ or 3 . We define co-index of $X$ as

$$
\text { co-ind }{ }_{G} X=\min \left\{k \mid \text { there exist an } G \text {-equivariant map } f: X \rightarrow \mathbb{S}^{(d+1) k+d}, k \geq 0\right\} .
$$

If no such $k$ exist then co-ind ${ }_{G} X=+\infty$. 
We define $\operatorname{ind}_{G} X=-1$ or co-ind ${ }_{G} X=-1$ if and only if $X=\emptyset$ where $G=\mathbb{S}^{1}$ or $\mathbb{S}^{3}$.

Now, we recall[11, 20] the mod 2 cohomology index of a paracompact Hausdorff free $G$-space, where $G=\mathbb{S}^{1}$ or $\mathbb{S}^{3}$. It is similar to the homology index for free involution on a paracompact Hausdorff space defined by Conner and Floyd[1].

Definition 3.3. Let $X$ be a free $G$-space, where $G=\mathbb{S}^{d}, d=1$ or 3 and $\omega \in H^{d+1}(X / G)$ be the Stiefel-Witney class of the associated $G$-bundle $X \rightarrow X / G$. Then the mod 2 cohomology $G$-index of $X$ is defined as the largest integer $k$ such that $\omega^{k} \neq 0$. It is deonoted by cohom-index ${ }_{G} X$.

Next, we observe that the index and co-index of $\mathbb{S}^{(d+1) n+d}$ for the action of componentwise multiplication of $\mathbb{S}^{d}$ is $n$, where $d=1$ or 3 .

Let $\mathbb{S}^{(d+1) n+d}$ be a free $\mathbb{S}^{d}$-space, $d=1$ or 3 , with the standard action. For all $k \leq n$, the inclusion map $i: \mathbb{S}^{(d+1) k+d} \rightarrow \mathbb{S}^{(d+1) n+d}$ is $\mathbb{S}^{d}$-equivariant, therefore, $\operatorname{ind}_{\mathbb{S}^{d}} \mathbb{S}^{(d+1) n+d} \geq k$. By the Borsuk-Ulam theorem, there does not exist an $\mathbb{S}^{d}$-equivariant map $f: \mathbb{S}^{(d+1) k+d} \rightarrow$ $\mathbb{S}^{(d+1) n+d}$ for $k>n$. This implies that $\operatorname{ind}_{\mathbb{S}^{d}} \mathbb{S}^{(d+1) n+d}=n$. By the similar argument, $\operatorname{co-ind}_{\mathbb{S}^{d}} \mathbb{S}^{(d+1) n+d}=n$.

We know that if $X$ is a finitistic free $G$-space, where $G=\mathbb{S}^{d}, d=1$ or 3 , with $\bmod 2$ cohomology ring of $\mathbb{S}^{(d+1) n+d}$ then the orbit space $X / G \sim_{2} \mathbb{F} \mathbb{P}^{n}$, where $\mathbb{F}=\mathbb{C}$ or $\mathbb{H}$. This gives that the mod 2 cohomology index of $X$ which admits free $G$ actions is $n([11])$.

Next, we observe some properties of the index and co-index of a $G$-space $X$ for $G=\mathbb{S}^{1}$ or $\mathbb{S}^{3}$ :

Theorem 3.4. Let $X$ and $Y$ be free $G$-spaces, where $G=\mathbb{S}^{d}, d=1$ or 3 .

(1) If $f: X \rightarrow Y$ is $G$-equivariant map then $\operatorname{ind}_{G} X \leq \operatorname{ind}_{G} Y$.

(2) If $f: X \rightarrow Y$ is $G$-equivariant map then co-ind ${ }_{G} X \leq$ co-ind $_{G} Y$.

(3) $\operatorname{ind}_{G} X \leq$ co-ind $_{G} X$.

(4) $\operatorname{co-ind}_{G}(X * Y) \leq \operatorname{co-ind}_{G} X+\operatorname{co-ind}_{G} Y+1$.

Proof. (1) and (2) follows from the definitions.

(3) Let $f: \mathbb{S}^{(d+1) k+d} \rightarrow X$ be any $\mathbb{S}^{d}$-equivariant map where $d=1$ or 3 . Then, we have $k=$ co-ind $_{\mathbb{S}^{d}} \mathbb{S}^{(d+1) k+d} \leq \operatorname{co-ind}_{\mathbb{S}^{d}} X$. Consequently, $\operatorname{ind}_{\mathbb{S}^{d}} X \leq \operatorname{co-ind}_{\mathbb{S}^{d}} X$.

(4) If either co-ind $\mathbb{S}_{\mathbb{S} d} X$ or co-ind $\mathbb{S}_{\mathbb{S}^{d}} Y$ are $+\infty$ then the result is trivially true. So, let co-ind $_{\mathbb{S}^{d}} X=m$ and co-ind $\mathbb{S}^{d} Y=n$. Let $f: X \rightarrow \mathbb{S}^{(d+1) m+d}$ and $g: Y \rightarrow \mathbb{S}^{(d+1) n+d}$ be the corresponding $\mathbb{S}^{d}$-equivariant maps. Then the map $f * g: X * Y \rightarrow \mathbb{S}^{(d+1) m+d} * \mathbb{S}^{(d+1) n+d} \approx$ $\mathbb{S}^{(d+1)(n+m+1)+d}$ defined by $f * g([x, y, t])=[f(x), g(y), t]$ is a $\mathbb{S}^{d}$-equivariant map. Hence, we get co-ind $\mathbb{S}^{d} X * Y \leq \operatorname{co-ind}_{\mathbb{S}^{d}} \mathbb{S}^{(d+1)(n+m+1)+d}=\operatorname{co-ind}_{\mathbb{S}^{d}} X+\operatorname{co-ind}_{\mathbb{S}^{d}} Y+1$.

Let $G=\mathbb{S}^{d}, d=1$ or 3 acts freely on $\mathbb{F}$ under the action of scalar multiplication, where $\mathbb{F}=\mathbb{C}$ or $\mathbb{H}$. Then, . : $\mathbb{F} \times G \rightarrow \mathbb{F}$ defined by $(w, c) \rightarrow c^{-1} . w$ is a free right $G$ action on $\mathbb{F}$. Let $\lambda=\left(\frac{X \times \mathbb{F}}{G}, q, X / G, \mathbb{F}\right)$ be the associated line bundle of the principal $G$-bundle 
$G \hookrightarrow X \stackrel{p}{\rightarrow} X / G$, where $q: \frac{X \times \mathbb{F}}{G} \rightarrow X / G$ is defined as $q([x, z])=p(x)=[x]$. As $E_{G}$ is free $G$-space, the map $h: X_{G} \rightarrow X / G$ defined by $h([x, e])=[x]$ is a fibre bundle associated to the principal $G$-bundle $G \hookrightarrow X \rightarrow X / G$. As $E_{G}$ is contractible, $h$ is a homotopy equivalence. Define $E_{1}(\gamma)=\left\{([v], t v) \in \mathbb{F P}^{\infty} \times \mathbb{F}^{\infty} \mid t \in \mathbb{F}\right\}$ and $r: E_{1}(\gamma) \rightarrow \mathbb{F P}^{\infty}$ is the restriction of projection map onto the first component. Then, $\xi=\left(E_{1}(\gamma), r, \mathbb{F} \mathbb{P}^{\infty}, \mathbb{F}\right)$ is a universal vector bundle of rank 1 .

Let $E$ denotes the total space of the pull back bundle $h^{*}(\lambda)$. We can define $\phi: E \rightarrow$ $E_{1}(\gamma)$ by $\phi\left([x, v],\left[x^{\prime}, c^{\prime}\right]\right)=\left([v], c^{\prime} \cdot \theta\left(x, x^{\prime}\right) \cdot v\right)$ where $\theta$ is the translation map of the principal $G$-bundle $p: X \rightarrow X / G$. For the Borel fibration $X \stackrel{i}{\hookrightarrow} X_{G} \stackrel{\pi}{\rightarrow} \mathbb{F} P^{\infty}$, it is easy to see that $\pi^{*}(\xi) \cong h^{*}(\lambda)$. So, we get the following lemma:

Lemma 3.5. Let $X$ be a free $G$-space, where $G=\mathbb{S}^{d}, d=1$ or 3 . Then the map $\pi \circ h^{\prime}: X / G \rightarrow \mathbb{F P}^{\infty}$ is a classifying map for the bundle $\lambda$ where $h^{\prime}: X / G \rightarrow X_{G}$ denotes a homotopy inverse of $h$.

For each $k \geq 1, \mathbb{F P}^{k} \subseteq \mathbb{F P}^{\infty}$. Put $\xi^{\prime}=\left.\xi\right|_{\mathbb{F}^{k}}$, the restriction bundle of the universal bundle $\xi$ at $\mathbb{C P}^{k}$. Note that $\xi^{\prime}=\left(r^{-1}\left(\mathbb{F P}^{k}\right), r, \mathbb{F P} \mathbb{P}^{k}, \mathbb{F}\right)$ is a tautological bundle of rank 1 , where $r^{-1}\left(\mathbb{F P}^{k}\right)=\left\{([z], t z) \in \mathbb{F P}^{k} \times \mathbb{F}^{k+1} \mid t \in \mathbb{F}\right\}$. It is easy to prove that

Lemma 3.6. Let $X$ be a free $G$-space, where $G=\mathbb{S}^{d}, d=1$ or 3 , and $f: \mathbb{S}^{(d+1) k+d} \rightarrow X$ be $G$-equivariant map, where $d=1$ or 3 . Then $\bar{f}^{*}(\lambda) \cong \xi^{\prime}$ where $\bar{f}: \mathbb{F P}^{k} \rightarrow X / G$ is a continuous map induced by $f$.

Now, we prove a Borsuk-Ulam type result for a finitistic space which admits free actions of $G=\mathbb{S}^{1}$ or $\mathbb{S}^{3}$.

Theorem 3.7. Let $X$ be a finitistic free $G$-space, where $G=\mathbb{S}^{d}, d=1$ or 3 . If the mod 2 cohomology index of $X$ is $n$, then there does not exist $G$-equivariant map $f: \mathbb{S}^{(d+1) k+d} \rightarrow$ $X$ for all $k>n$, where $\mathbb{S}^{(d+1) k+d}$ equipped with action of componentwise multiplication of $\mathbb{S}^{d}$.

Proof. We prove for $G=\mathbb{S}^{3}$. Suppose, on the contrary, that there exists a $G$-equivariant map $f: \mathbb{S}^{4 k+3} \rightarrow X$ for $k>n$. Then $f$ induces a continuous map $\bar{f}: \mathbb{H}^{k} \rightarrow X / G$ and let $p: X \rightarrow X / G$ be the principal $G$-bundle. Note that the Borel fibration $X \stackrel{i}{\hookrightarrow}$ $X_{G} \stackrel{\pi}{\rightarrow} \mathbb{H} \mathbb{P}^{\infty}$ is a fibre bundle with structure group $G$. Let $h: X_{G} \rightarrow X / G$ be homotopy equivalence and $\xi=\left(E_{1}(\gamma), \gamma, \mathbb{H}^{\infty}, \mathbb{H}\right)$ be universal quaternion vector bundle of rank 1 , where $E_{1}(\gamma)=\left\{([v], t v) \in \mathbb{H}^{\infty} \times \mathbb{H}^{\infty} \mid t \in \mathbb{H}\right\}$ and $\gamma: E_{1}(\gamma) \rightarrow \mathbb{H}^{\infty}, \gamma([v], t v)=[v]$ be the projection map. Treat it as a real vector bundle of rank 4 . Let $\xi^{\prime}$ be the restriction bundle of $\xi$ at $\mathbb{H}^{k}$. By Lemma 3.5, $\pi \circ h^{\prime}: X / G \rightarrow \mathbb{H} \mathbb{P}^{\infty}$ is a classifying map for associated line bundle $\lambda=\left(\frac{X \times \mathbb{H}}{G}, q, X / G, \mathbb{H}\right)$ of principal $G$-bundle $p: X \rightarrow X / G$, where $h^{\prime}$ denotes the homotopy inverse of $h$. We know that $H^{*}\left(\mathbb{H} \mathbb{P}^{\infty}\right)=\mathbb{Z}_{2}[t]$, where $\operatorname{deg} t=4$. So the first nonzero Steifel-Witney class of $\xi$ is $\omega_{4}(\xi)=t$. Put $\pi^{*}(t)=u$. By the naturality of Witney 
classes, we get $\left(h^{\prime}\right)^{*}(u)=\left(h^{\prime}\right)^{*}\left(\pi^{*}(t)\right)=\left(\pi \circ h^{\prime}\right)^{*}\left(\omega_{4}(\xi)\right)=\omega_{4}(\lambda)$. As $f$ is equivariant map, by Lemma 3.6. we get $\xi^{\prime} \cong \bar{f}^{*}(\lambda)$ as real vector bundles. Again by the naturality of Witney classes,

$$
\bar{f}^{*}\left(\omega_{4}(\lambda)\right)=\omega_{4}\left(\bar{f}^{*}(\lambda)\right)=\omega_{4}\left(\xi^{\prime}\right)=t^{\prime}, \text { say. }
$$

So, $\left(h^{\prime}\right)^{*}(u)=\omega_{4}(\lambda)$ and $\bar{f}^{*}\left(\omega_{4}(\lambda)\right)=t^{\prime} \neq 0$. So, we get $t^{\prime}=\bar{f}^{*}\left(\left(h^{\prime}\right)^{*}(u)\right)$ and hence $t^{\prime}$ is the generator of $H^{4}\left(\mathbb{H} \mathbb{P}^{k}\right)$. Note that $\left(h^{\prime}\right)^{*}(u)$ is the characteristic class for the principal bundle $X \rightarrow X / G$. By our hypothesis, the mod 2 cohomology index of $X$ is $n$ which gives that $\left(\left(h^{\prime}\right)^{*}(u)\right)^{n+1}=0$. So, we get $0=\bar{f}^{*}\left(\left(\left(h^{\prime}\right)^{*}(u)\right)^{n+1}\right)=t^{\prime n+1}$ which contradicts that $t^{\prime}$ is the generator of $H^{4}\left(\mathbb{H}^{k}\right)$.

Similarly, we can prove for $G=\mathbb{S}^{1}$.

From the above theorem, it is easy to derive

Corollary 3.8. Let $X$ be a finitistic free $G$-space, where $G=\mathbb{S}^{d}, d=1$ or 3 . Then

$$
\operatorname{ind}_{G} X \leq \text { cohom-ind }_{G} X .
$$

\section{4. $\mathbb{S}^{1}$ AND $\mathbb{S}^{3}$ ACTIONS ON PRODUCT OF SPHERES}

In this section, we will compute the cohomology structure of the orbit spaces of free actions of $G=\mathbb{S}^{1}$ or $\mathbb{S}^{3}$ on a finitistic space $X \sim_{R} \mathbb{S}^{n} \times \mathbb{S}^{m}, 1 \leq n \leq m$, where $R=\mathbb{Q}$ or $\mathbb{Z}_{2}$. We also obtain an upper bound of the index of $X \sim_{\mathbb{Z}_{2}} \mathbb{S}^{n} \times \mathbb{S}^{m}$ for free $G$ actions and establish Borsuk-Ulam type results. By the Kunneth formula, we have $H^{*}(X)=$ $R[x, y] /\left\langle x^{2}, y^{2}\right\rangle, \quad \operatorname{deg} x=n, \operatorname{deg} y=m$.

First, we discuss free actions of $G=\mathbb{S}^{3}$ on $X$ with the rational coefficients.

Theorem 4.1. Let $G=\mathbb{S}^{3}$ acts freely on a finitistic space $X \sim_{\mathbb{Q}} \mathbb{S}^{n} \times \mathbb{S}^{m}, 1 \leq n \leq m$. Then $H^{*}(X / G)$ is isomorphic to one of the following:

(i) $\mathbb{Q}[u, v]\left\langle u^{\frac{n+1}{4}}, v^{2}\right\rangle$, where $\operatorname{deg} u=4, \operatorname{deg} v=m, n \equiv 3(\bmod 4)$

(ii) $\mathbb{Q}[u, v] /\left\langle u^{\frac{m+n+1}{4}}, u^{\frac{m-n+1}{4}} v-\alpha u^{\frac{m+1}{4}}, v^{2}-\beta u^{\frac{n}{2}}-\gamma u^{\frac{n}{4}} v\right\rangle$, where $\operatorname{deg} u=4, \operatorname{deg} v=$ $n, \alpha, \beta, \gamma \in \mathbb{Q}, m-n \equiv 3(\bmod 4), n$ is even, $m$ is odd and $\alpha=0$ if $m<2 n$ or $n \equiv 2(\bmod 4)$ and $\gamma=0$ if $n \not \equiv 0(\bmod 4)$

(iii) $\mathbb{Q}[u, v] /\left\langle u^{\frac{m+1}{4}}, v^{2}-\alpha u^{\frac{n}{2}}-\beta u^{\frac{n}{4}} v\right\rangle$, where $\operatorname{deg} u=4, \operatorname{deg} v=n, m \equiv 3(\bmod 4), \alpha, \beta \in$ $\mathbb{Q}, \beta=0$ if $n \not \equiv 0(\bmod 4)$ and $\alpha=0$ if either $n \not \equiv 2(\bmod 4)$ or $2 n \geq m$.

Proof. As $\pi_{1}\left(B_{G}\right)$ acts trivially on $X, E_{2}^{k, l} \cong H^{k}\left(B_{G}\right) \otimes H^{l}(X)$. First, we assume $n<m$. The possible nontrivial differentials are $d_{n+1}, d_{m+1}, d_{m-n+1}$ or $d_{m+n+1}$. There are two possible cases: (i) $d_{r}(1 \otimes x) \neq 0$, and (ii) $d_{r}(1 \otimes x)=0$ and $d_{r}(1 \otimes y) \neq 0$.

Case (i): When $d_{r}(1 \otimes x) \neq 0$, then $r$ must be $n+1, n \equiv 3(\bmod 4)$ and $d_{n+1}(1 \otimes x)=$ $c t^{\frac{n+1}{4}} \otimes 1$ for some $0 \neq c \in \mathbb{Q}$. If $m=2 n$ and $d_{n+1}(1 \otimes y)=d t^{\frac{n+1}{4}} \otimes x$, where $0 \neq d \in \mathbb{Q}$, then $0=d_{n+1}\left(1 \otimes y^{2}\right)=2 d t^{\frac{n+1}{4}} \otimes x y$ which is not possible. Therefore, $d_{n+1}(1 \otimes y)=0$. Consequently, $d_{n+1}\left(t^{k} \otimes x\right)=c t^{k+\frac{n+1}{4}} \otimes 1, d_{n+1}\left(t^{k} \otimes y\right)=0$ and $d_{n+1}\left(t^{k} \otimes x y\right)=c t^{k+\frac{n+1}{4}} \otimes y$ 
for all $k \geq 0$, and $E_{2}^{*, *}=E_{n+1}^{*, *}$. This implies that $d_{n+1}: E_{n+1}^{k, l} \rightarrow E_{n+1}^{k+n+1, l-n}$ is an isomorphism for all $k \geq 0$, and $l=n$ or $n+m$. So, we get $E_{n+2}^{k, l}=0$ for all $k \geq 0, l=n$ or $n+m$; and $E_{n+2}^{k, l}=E_{2}^{k, l}$ if $k<n+1, l=0$ or $m$ and trivial otherwise. As $n<m, d_{m+1}$ is the trivial homomorphism and so $E_{\infty}^{*, *}=E_{n+2}^{*, *}$. So, we have

$$
H^{j}\left(X_{G}\right)= \begin{cases}\mathbb{Q} & \text { if } 0 \leq j \equiv 0(\bmod 4)<n, m \leq j \equiv m(\bmod 4)<m+n \\ 0 & \text { otherwise. }\end{cases}
$$

Note that $t \otimes 1 \in E_{2}^{4,0}$ and $1 \otimes y \in E_{2}^{0, m}$ are permanent cocyles, where $t$ generates $H^{*}\left(B_{G}\right)$. Let $w \in E_{\infty}^{0, m}$ and $u \in E_{\infty}^{4,0}$ be elements corresponding to $1 \otimes y \in E_{2}^{0, m}$ and $t \otimes 1 \in E_{2}^{4,0}$, respectively. Then $w^{2}=0$ and $u^{\frac{n+1}{4}}=0$. Therefore, there exist $v \in H^{m}\left(X_{G}\right)$ such that $i^{*}(v)=y$. So, we have $v^{2}=0$. Thus,

$$
H^{*}\left(X_{G}\right)=\frac{\mathbb{Q}[u, v]}{\left\langle u^{\frac{n+1}{4}}, v^{2}\right\rangle}
$$

where $\operatorname{deg} u=4, \operatorname{deg} v=m$. This realizes case(i).

Case(ii): When $d_{r}(1 \otimes x)=0$ and $d_{r}(1 \otimes y) \neq 0$. Then, there are two subcases:

Subcase(i): When $r=m-n+1$.

In this case, $m-n \equiv 3(\bmod 4)$ and $d_{m-n+1}(1 \otimes y)=c t^{\frac{m-n+1}{4}} \otimes x$ for some $0 \neq c \in \mathbb{Q}$. If $m$ is even then $0=d_{m-n+1}\left(1 \otimes y^{2}\right)=2 c t^{\frac{m-n+1}{4}} \otimes x y$ which is not possible. Therefore, $m$ must be odd and $n$ is even. We get $d_{m-n+1}\left(t^{k} \otimes x y\right)=0, d_{m-n+1}\left(t^{k} \otimes y\right)=c t^{k+\frac{m-n+1}{4}} \otimes x$ for all $k \geq 0$, and $E_{2}^{*, *}=E_{m-n+1}^{*, *}$. This implies that $d_{m-n+1}: E_{m-n+1}^{k, m} \rightarrow E_{m-n+1}^{k+m-n+1, n}$ is an isomorphism for all $k \geq 0$. So, $E_{m-n+2}^{k, l}=E_{2}^{k, l}$ for all $k \geq 0, l=0, n+m ; E_{m-n+2}^{k, n}=E_{2}^{k, n}$ if $k<m-n+1$ and trivial otherwise. As $G$ acts freely on $X, d_{m+n+1}$ must be nontrivial. Let $d_{m+n+1}(1 \otimes x y)=d t^{\frac{m+n+1}{4}} \otimes 1$, where $0 \neq d \in \mathbb{Q}$. Then, $d_{m+n+1}\left(t^{k} \otimes x y\right)=d t^{k+\frac{m+n+1}{4}} \otimes 1$ for all $k \geq 0$, and so $d_{m+n+1}: E_{m+n+1}^{k, m+n} \rightarrow E_{m+n+1}^{k+m+n+1,0}$ is an isomorphism for all $k \geq 0$. This implies that $E_{m+n+2}^{k, n}=E_{m-n+2}^{k, n}, E_{m+n+2}^{k, m+n}=0$ for all $k \geq 0$, and $E_{m+n+2}^{k, 0}=E_{m-n+2}^{k, 0}$ if $k<m+n+1$ and trivial otherwise. Now, $E_{\infty}^{*, *}=E_{m+n+2}^{*, *}$. If $n \equiv 0(\bmod 4)$ then $m \equiv 3(\bmod 4)$, and we get

$$
H^{j}(X / G)= \begin{cases}\mathbb{Q} & \text { if } 0 \leq j \equiv 0(\bmod 4)<n, m<j \equiv 0(\bmod 4)<n+m \\ \mathbb{Q} \oplus \mathbb{Q} & \text { if } n \leq j \equiv 0(\bmod 4)<m \\ 0 & \text { otherwise. }\end{cases}
$$

If $n \equiv 2(\bmod 4)$ then $m \equiv 1(\bmod 4)$, and we get

$$
H^{j}(X / G)= \begin{cases}\mathbb{Q} & \text { if } 0 \leq j \equiv 0(\bmod 4)<n+m, n \leq j \equiv 2(\bmod 4)<m \\ 0 & \text { otherwise. }\end{cases}
$$

Let $w \in E_{\infty}^{0, n}$ and $u \in E_{\infty}^{4,0}$ be elements corresponding to permanent cocycles $1 \otimes x \in$ $E_{2}^{0, n}$ and $t \otimes 1 \in E_{2}^{4,0}$, respectively. We have $w^{2}=u^{\frac{m+n+1}{4}}=u^{\frac{m-n+1}{4}} w=0$. This implies that

$$
\operatorname{Tot} E_{\infty}^{*, *} \cong \frac{\mathbb{Q}[u, w]}{\left\langle w^{2}, u^{\frac{m+n+1}{4}}, u^{\frac{m-n+1}{4}} w\right\rangle}, \text { where deg } u=4 \text { and } \operatorname{deg} w=n
$$


There exist $v \in H^{n}\left(X_{G}\right)$ corresponding to $w \in E_{\infty}^{0, n}$ such that $i^{*}(v)=x$. As $u^{\frac{m-n+1}{4}} w=0$ in $\operatorname{Tot} E_{\infty}^{*, *}$, we get $u^{\frac{m-n+1}{4}} v=\alpha u^{\frac{m+1}{4}}, \alpha \in \mathbb{Q}$ and $\alpha=0$ when $n<2 m$ or $n \equiv 2(\bmod 4)$. Also, if $2 n \leq m+n-3$ then $u^{\frac{n}{2}} \in H^{2 n}\left(X_{G}\right)$ and if $n$ is multiple of 4 then $u^{\frac{n}{4}} v \in H^{2 n}\left(X_{G}\right)$. Therefore, $v^{2}=\alpha u^{\frac{n}{2}}+\beta u^{\frac{n}{4}} v$ where $\alpha, \beta \in \mathbb{Q}$. Thus, we have

$$
H^{*}\left(X_{G}\right)=\frac{\mathbb{Q}[u, v]}{\left\langle u^{\frac{m+n+1}{4}}, u^{\frac{m-n+1}{4}} v-\alpha u^{\frac{m+1}{4}}, v^{2}-\beta u^{\frac{n}{2}}-\gamma u^{\frac{n}{4}} v\right\rangle}
$$

where $\operatorname{deg} u=4, \operatorname{deg} v=n, \alpha, \beta, \gamma \in \mathbb{Q}, m-n \equiv 3(\bmod 4), n$ is even, $m$ is odd and $\alpha=0$ if $m<2 n$ or $n \equiv 2(\bmod 4)$ and $\gamma=0$ if $n \not \equiv 0(\bmod 4)$. This realizes case(ii) of the theorem.

Subcase (ii): When $r=m+1$.

In this case, $m \equiv 3(\bmod 4)$ and $d_{n+1}=d_{m-n+1} \equiv 0$. Let $d_{m+1}(1 \otimes y)=c t^{\frac{m+1}{4}} \otimes 1$ for some $0 \neq c \in \mathbb{Q}$. Then $d_{m+1}(1 \otimes x)=0$ and $d_{m+1}\left(t^{k} \otimes x y\right)=(-1)^{n} c t^{k+\frac{m+1}{4}} \otimes y$ for all $k \geq 0$ and $E_{2}^{*, *}=E_{m+1}^{*, *}$. This implies that $d_{m+1}: E_{m+1}^{k, l} \rightarrow E_{m+1}^{k+m+1, l-m}$ is an isomorphism for all $k \geq 0$, and $l=m$ or $m+n$. So, we get $E_{m+2}^{k, l}=E_{2}^{k, l}$ if $k<m+1$ and $l=0$ or $n$ and trivial otherwise. So, $E_{\infty}^{*, *}=E_{m+2}^{*, *}$. If $n \equiv 0(\bmod 4)$, then

$$
H^{j}\left(X_{G}\right)= \begin{cases}\mathbb{Q} & \text { if } 0 \leq j \equiv 0(\bmod 4)<n, m<j \equiv 0(\bmod 4)<m+n \\ \mathbb{Q} \oplus \mathbb{Q} & \text { if } n \leq j \equiv 0(\bmod 4)<m \\ 0 & \text { otherwise. }\end{cases}
$$

If $n \not \equiv 0(\bmod 4)$, then

$$
H^{j}\left(X_{G}\right)= \begin{cases}\mathbb{Q} & \text { if } 0 \leq j \equiv 0(\bmod 4)<m, n \leq j \equiv n(\bmod 4)<n+m \\ 0 & \text { otherwise. }\end{cases}
$$

Let $w \in E_{\infty}^{0, n}$ and $u \in E_{\infty}^{4,0}$ be elements corresponding to permanent cocycles $1 \otimes x \in E_{2}^{0, n}$ and $t \otimes 1 \in E_{2}^{4,0}$, respectively. Then $w^{2}=0$ and $u^{\frac{m+1}{4}}=0$. There exist $v \in H^{n}\left(X_{G}\right)$ corresponding to $w \in E_{\infty}^{0, n}$ such that $i^{*}(v)=x$. We have $v^{2}=\alpha u^{\frac{n}{2}}+\beta u^{\frac{n}{4}} v$, where $\alpha, \beta \in \mathbb{Q}$. Thus,

$$
H^{*}\left(X_{G}\right)=\frac{\mathbb{Q}[u, v]}{\left\langle u^{\frac{m+1}{4}}, v^{2}-\alpha u^{\frac{n}{2}}-\beta u^{\frac{n}{4}} v\right\rangle}
$$

where $\operatorname{deg} u=4, \operatorname{deg} v=n, \alpha, \beta \in \mathbb{Q}, \beta=0$ if $n \not \equiv 0(\bmod 4)$ and $\alpha=0$ if either $n \not \equiv$ $2(\bmod 4)$ or $2 n \geq m$. This realizes case(iii) of the theorem.

Now, we assume $n=m$. If $d_{n+1}(1 \otimes x)=c t^{\frac{n+1}{4}} \otimes 1$ and $d_{n+1}(1 \otimes y)=0$ for some $0 \neq c \in \mathbb{Q}$, then it is same as case(i). Now, suppose $d_{n+1}(1 \otimes x)=c t^{\frac{n+1}{4}} \otimes 1$ and $d_{n+1}(1 \otimes y)=d t^{\frac{n+1}{4}} \otimes 1$ for some $0 \neq c, d \in \mathbb{Q}$. Then, $n \equiv 3(\bmod 4), d_{n+1}\left(1 \otimes\left(c_{1} x+c_{2} y\right)\right)=$ $t^{\frac{n+1}{4}} \otimes\left(c_{1} c+c_{2} d\right), d_{n+1}(1 \otimes x y)=t^{\frac{n+1}{4}} \otimes(c y-d x)$ and $E_{2}^{*, *}=E_{n+1}^{*, *}$. This implies that $d_{n+1}: E_{n+1}^{k, 2 n} \rightarrow E_{n+1}^{k+n+1, n}$ is injective; and $\operatorname{ker}\left(d_{n+1}: E_{n+1}^{4 k, n} \rightarrow E_{n+1}^{4 k+n+1,0}\right) \cong \mathbb{Q}$ and $\operatorname{im}\left(d_{n+1}: E_{n+1}^{4 k-n-1,2 n} \rightarrow E_{n+1}^{4 k, n}\right) \cong \mathbb{Q}$ with basis $\left\{t^{k} \otimes(c y-d x)\right\}$ and $\left\{t^{k-\frac{n+1}{4}} \otimes(c y-d x)\right\}$, respectively, for all $k \geq 0$. So, we get $E_{n+2}^{4 k, 0}=E_{n+2}^{4 k, n} \cong \mathbb{Q}$ with basis $\left\{t^{k} \otimes 1\right\}$ and $\left\{t^{k} \otimes(c y-d x)\right\}$, respectively, for all $0 \leq k<\frac{n+1}{4}$; and trivial otherwise. So, $E_{\infty}^{*, *}=E_{n+2}^{*, *}$. 
So, we have

$$
H^{j}\left(X_{G}\right)= \begin{cases}\mathbb{Q} & \text { if } 0 \leq j \equiv 0(\bmod 4)<n, n \leq j \equiv n(\bmod 4)<2 n \\ 0 & \text { otherwise. }\end{cases}
$$

Let $w \in E_{\infty}^{0, n}$ and $u \in E_{\infty}^{4,0}$ be elements corresponding to permanent cocycles $1 \otimes(c y-d x) \in$ $E_{2}^{0, n}$ and $t \otimes 1 \in E_{2}^{4,0}$, respectively. Then $w^{2}=0$ and $u^{\frac{n+1}{4}}=0$. There exist $v \in H^{n}\left(X_{G}\right)$ corresponding to $w \in E_{\infty}^{0, n}$ such that $i^{*}(v)=c y-d x$. We have $v^{2}=0$, and thus

$$
H^{*}\left(X_{G}\right)=\frac{\mathbb{Q}[u, v]}{\left\langle u^{\frac{n+1}{4}}, v^{2}\right\rangle}
$$

where $\operatorname{deg} u=4, \operatorname{deg} v=n$. This realizes case(i) of the theorem.

Now, we discuss free actions of $G=\mathbb{S}^{3}$ on $X \sim_{2} \mathbb{S}^{n} \times \mathbb{S}^{m}$. First, we derive following results.

Lemma 4.2. Let $X$ be a finitistic free $G$-space with $X \sim_{2} \mathbb{S}^{n} \times \mathbb{S}^{m}, 1 \leq n \leq m$, where $G=\mathbb{S}^{d}, d=1$ or 3 . Then $H^{i}(X / G)=0$ for all $i \geq n+m-(d-1)$.

Proof. By Proposition 2.2, $H^{i}(X / G)=0$ for all $i>n+m$. For $n+m-(d-1) \leq i \leq n+m$, the result follows by taking $n+m+1 \leq k \leq n+m+d$ in the Gysin sequence of the sphere bundle $G \hookrightarrow X \rightarrow X / G$.

Lemma 4.3. Let $X$ be a finitistic free $G$-space with $X \sim_{2} \mathbb{S}^{n} \times \mathbb{S}^{m}, 1 \leq n \leq m$, and $p: X \rightarrow X / G$ be the orbit map, where $G=\mathbb{S}^{d}, d=1$ or 3 . Then $p^{*}: H^{i}(X / G) \rightarrow H^{i}(X)$ cannot be nontrivial for both $i=n$ and $m$.

Proof. Assume otherwise. Then, there exist nonzero elements $u \in H^{n}(X / G)$ and $v \in$ $H^{m}(X / G)$ such that $p^{*}(u)=x$ and $p^{*}(v)=y$. Consequently, $p^{*}(u v)=x y \neq 0$ in $H^{n+m}(X)$. This implies that $u v \neq 0$ in $H^{n+m}(X / G)$, a contradiction.

Now, we determine the orbit spaces of a finitistic space $X \sim_{2} \mathbb{S}^{n} \times \mathbb{S}^{m}, 1 \leq n \leq m$ for $G=\mathbb{S}^{d}, d=1$ or 3 , actions.

Theorem 4.4. Let $X$ be a finitistic free $G$-space with $X \sim_{2} \mathbb{S}^{n} \times \mathbb{S}^{m}, 1 \leq n \leq m$, where $G=\mathbb{S}^{3}$. Then $H^{*}(X / G)$ is isomorphic to one of the following graded algebras:

(i) $\mathbb{Z}_{2}[u, v] /\left\langle u^{\frac{m+1}{4}}, v^{2}+\alpha v u^{\frac{n}{4}}+\beta u^{\frac{n}{2}}\right\rangle$, where $\operatorname{deg} u=4, \operatorname{deg} v=n, m \equiv 3(\bmod 4)$, $\beta=0$ if $m<2 n$ and $n$ is even; and $\alpha=\beta=0$ if $n$ is odd.

(ii) $\mathbb{Z}_{2}[u, v] /\left\langle u^{\frac{n+m+1}{4}}, v u^{\frac{m-n+1}{4}}, v^{2}+\alpha v u^{\frac{n}{4}}+\beta u^{\frac{n}{2}}\right\rangle$, where $\operatorname{deg} u=4, \operatorname{deg} v=n, m-n \equiv$ $3(\bmod 4), m \equiv 3(\bmod 4)$ and $\alpha=0$ if $m<2 n$.

(iii) $\mathbb{Z}_{2}[u, v] /\left\langle u^{\frac{n+1}{4}}, v^{2}\right\rangle$, where $\operatorname{deg} u=2, \operatorname{deg} v=m$ and $n \equiv 3(\bmod 4)$.

Proof. Recall that the Gysin sequence of the sphere bundle $G \hookrightarrow X \stackrel{p}{\longrightarrow} X / G$ is:

$$
\cdots \longrightarrow H^{i}(X) \stackrel{\rho}{\longrightarrow} H^{i-3}(X / G) \stackrel{\cup}{\longrightarrow} H^{i+1}(X / G) \stackrel{p^{*}}{\longrightarrow} H^{i+1}(X) \stackrel{\rho}{\longrightarrow} H^{i-2}(X / G) \longrightarrow \cdots
$$


which begins with

$$
0 \longrightarrow H^{3}(X / G) \stackrel{p *}{\longrightarrow} H^{3}(X) \stackrel{\rho}{\longrightarrow} H^{0}(X / G) \stackrel{\cup}{\longrightarrow} H^{4}(X / G) \stackrel{p^{*}}{\longrightarrow} H^{4}(X) \longrightarrow \cdots
$$

Then, for $0<i<n-1, n<i<m-1$ and $m<i<n+m-1$, we have $H^{i-3}(X / G) \cong$ $H^{i+1}(X / G)$. We also have $H^{i}(X / G) \cong H^{i}(X)$ for $0 \leq i \leq 2$. This gives that $\mathbb{Z}_{2} \cong$ $H^{i}(X / G) \cong H^{i+4}(X / G)$ for $0 \leq i \equiv 0(\bmod 4)<n-4$ and $H^{i}(X / G)=0$ for $0<i \equiv$ $j(\bmod 4)<n$, where $1 \leq j \leq 3$. Let $u \in H^{4}(X / G)$ be the image of the generator $1 \in H^{0}(X / G)$ under the homomorphism $\cup: H^{0}(X / G) \rightarrow H^{4}(X / G)$. So, inductively generator of $H^{i}(X / G)$ is $\left\{u^{\frac{i}{4}}\right\}$ for all $0 \leq i \equiv 0(\bmod 4)<n$. As $\mathbb{S}^{3}$ acts freely on $X$, both $n$ and $m$ cannot be even [5]. It is clear that isomorphisms appears in differences of mod 4, so we consider the following cases:

Case(I): When $n \equiv 1(\bmod 4)$.

As $n \equiv 1(\bmod 4), H^{n-1}(X / G) \cong \mathbb{Z}_{2}$ with the basis $\left\{u^{\frac{n-1}{4}}\right\}$ and $H^{n-j}(X / G)=0$ for $2 \leq j \leq 4$. This implies that $H^{n+1}(X / G)=H^{n+2}(X / G)=0, H^{n}(X / G) \cong H^{n}(X)$ and $H^{n+3}(X / G) \cong H^{n-1}(X / G)$. So the basis for $H^{n}(X / G)$ and $H^{n+3}(X / G)$ are $\{v\}$ and $\left\{u^{\frac{n+3}{4}}\right\}$, respectively where $p^{*}(v)=x$. Consequently, $H^{i}(X / G) \cong \mathbb{Z}_{2}$ for $n \leq i \equiv j(\bmod$ $4)<m, j=0$ or 1 ; and $H^{i}(X / G)=0$ for $n<i \equiv j(\bmod 4)<m, j=2$ or 3 . Inductively, the bases for $H^{i}(X / G)$ are $\left\{v u^{\frac{i-n}{4}}\right\}$ when $n \leq i \equiv 1(\bmod 4)<m$, and $\left\{u^{\frac{i}{4}}\right\}$ when $n \leq i \equiv 0(\bmod$ $4)<m$. Now, the possible values of $m$ are as (a) $m \equiv 3(\bmod 4)$, (b) $m \equiv 2(\bmod 4)$, (c) $m \equiv 1(\bmod 4)$ and $(d) m \equiv 0(\bmod 4)$.

Subcase(a): As $m \equiv 3(\bmod 4)$, we get $0=H^{m-1}(X / G)=H^{m-4}(X / G)$, and $\mathbb{Z}_{2} \cong$ $H^{m-2}(X / G) \cong H^{m-3}(X / G)$. By Lemma 4.3, $p^{*}: H^{m}(X / G) \rightarrow H^{m}(X)$ must be trivial. This implies that $H^{m+2}(X / G) \cong H^{m-2}(X / G), H^{m+3}(X / G)=H^{m}(X / G)=0$ and $\rho$ : $H^{m}(X) \rightarrow H^{m-3}(X / G)$ is an isomorphism. Consequently, $H^{m+1}(X / G)=0$. We have $H^{i-3}(X / G) \cong H^{i+1}(X / G)$ for $m<i<n+m-1$. So, we get $H^{m+n-j}(X / G)=0$ for $j=1,2$ and $4 ;$ and $H^{m+n-3}(X / G) \cong \mathbb{Z}_{2}$. Accordingly, we get

$$
H^{i}(X / G)= \begin{cases}\mathbb{Z}_{2} & \text { if } 0 \leq i \equiv 0(\bmod 4)<m, n \leq i \equiv 1(\bmod 4)<n+m \\ 0 & \text { otherwise }\end{cases}
$$

If $m=n+2$ then we get similar cohomology groups. Note that the basis of $H^{m-3}(X / G)$ is $\left\{u^{\frac{m-3}{4}}\right\}$. As the homomorphism $\cup: H^{m-3}(X / G) \rightarrow H^{m+1}(X / G)$ is trivial, we get $u^{\frac{m+1}{4}}=$ 0 . Since $n \equiv 1(\bmod 4)$, we get $H^{2 n}(X / G)=0$. Consequently, $v^{2}=0$. Therefore, $H^{*}(X / G)$ is given by $\mathbb{Z}_{2}[u, v] /\left\langle u^{\frac{m+1}{4}}, v^{2}\right\rangle$ where $\operatorname{deg} u=4, \operatorname{deg} v=n$. This realizes possibility (i).

Subcase(b): As $m \equiv 2(\bmod 4)$, we get $H^{m-4}(X / G)=H^{m-3}(X / G)=0$ and $H^{m-1}(X / G)$ $\cong H^{m-2}(X / G) \cong \mathbb{Z}_{2}$. By Lemma 4.3, $p^{*}: H^{m}(X / G) \rightarrow H^{m}(X)$ must be trivial. This implies that $H^{m}(X / G)=H^{m+1}(X / G)=0, H^{m+2}(X / G) \cong H^{m-2}(X / G)$ and $H^{m+3}(X / G) \cong H^{m-1}(X / G)$. Consequently, $H^{m+n-2}(X / G) \cong \mathbb{Z}_{2}$, which contradicts that $G$ acts freely on $X$. If $m=n+1$ then we get same cohomology groups.

Subcase(c): When $m \equiv 1(\bmod 4)$. If $n \neq m$ then we get $H^{m-3}(X / G)=H^{m-2}(X / G)$ $=0$ and $H^{m-4}(X / G) \cong H^{m-1}(X / G) \cong \mathbb{Z}_{2}$. By the exactness of the Gysin sequence, 
$p^{*}: H^{m}(X / G) \rightarrow H^{m}(X)$ must be nontrivial, a contradiction. If $n=m$ then $H^{n}(X / G) \cong$ $H^{n}(X) \cong \mathbb{Z}_{2} \oplus \mathbb{Z}_{2}$ and $H^{n+j}(X / G)=0$ for $1 \leq j \leq 3$. This gives $H^{2 n-1}(X / G) \cong \mathbb{Z}_{2} \oplus \mathbb{Z}_{2}$, a contradiction.

Subcase(d): As $m \equiv 0(\bmod 4)$, we get $H^{m-1}(X / G)=H^{m-2}(X / G)=0$ and $H^{m-4}(X / G)$ $\cong H^{m-3}(X / G) \cong \mathbb{Z}_{2}$. By Lemma 4.3, $p^{*}: H^{m}(X / G) \rightarrow H^{m}(X)$ must be trivial. This implies that $H^{m}(X / G) \cong \mathbb{Z}_{2}$. Consequently, $H^{m+n-1}(X / G) \cong \mathbb{Z}_{2}$ which contradicts that $G$ acts freely on $X$. If $m=n+3$ then we get the same cohomology groups.

Case(II): When $n \equiv 3(\bmod 4)$. First, we consider $n \neq m$.

As $n \equiv 3(\bmod 4)$, we get $H^{n-3}(X / G) \cong \mathbb{Z}_{2}$ with the basis $\left\{u^{\frac{n-3}{4}}\right\}$ and $H^{n-j}(X / G)$

$=0$ for $j=1,2,4$. By the exactness of the Gysin sequence, $H^{n+j}(X / G)=0$ for $j=2$ and 3. There are two cases:

Subcase(i): When $p^{*}: H^{n}(X / G) \rightarrow H^{n}(X)$ is trivial.

In this case, $\rho: H^{n}(X) \rightarrow H^{n-3}(X / G)$ is an isomorphism and $H^{n+j}(X / G)=0$ for $j=0,1$. Accordingly, $H^{i}(X / G)=0$ for $n \leq i<m$. By the exactness of the Gysin sequence, $H^{m}(X / G) \cong H^{m}(X)$ and $H^{m+j}(X / G)=0$ for $1 \leq j \leq 3$. Consequently, $H^{m+n-3}(X / G) \cong \mathbb{Z}_{2}$ and $H^{m+n-j}(X / G)=0$ for $j=1,2$ and 4 . Thus, we have

$$
H^{i}(X / G)= \begin{cases}\mathbb{Z}_{2} & \text { if } 0 \leq i \equiv 0(\bmod 4)<n, m \leq i \equiv m(\bmod 4)<n+m \\ 0 & \text { otherwise }\end{cases}
$$

As $\cup: H^{n-3}(X / G) \rightarrow H^{n+1}(X / G)$ is trivial, we get $u^{\frac{n+1}{4}}=0$. Since $p^{*}: H^{m}(X / G) \rightarrow$ $H^{m}(X)$ is an isomorphism, we have $p^{*}(v)=y$ where $\{v\}$ is the basis of $H^{m}(X / G)$. Now $n<m$ implies that $v^{2}=0$. Therefore, $H^{*}(X / G)$ is given by $\mathbb{Z}_{2}[u, v] /\left\langle u^{\frac{n+1}{4}}, v^{2}\right\rangle$ where $\operatorname{deg} u=4, \operatorname{deg} v=m$. This realizes possibility (iii).

Subcase(ii): When $p^{*}: H^{n}(X / G) \rightarrow H^{n}(X)$ is nontrivial.

Then $H^{n}(X / G) \cong H^{n}(X)$ and $H^{n+1}(X / G) \cong H^{n-3}(X / G)$. From this we get, $H^{i}(X / G)$ $=0$ for $n<i \equiv j(\bmod 4)<m, j=1$ or 2 and $H^{i}(X / G) \cong \mathbb{Z}_{2}$ for $n \leq i \equiv j(\bmod 4)<m$, $j=0$ or 3. Inductively, the bases for $H^{i}(X / G)$ are $\left\{u^{\frac{i}{4}}\right\}$ when $n<i \equiv 0(\bmod 4)<m$, and $\left\{v u^{\frac{i-n}{4}}\right\}$ when $n \leq i \equiv 3(\bmod 4)<m$ where $v \in H^{n}(X / G)$ such that $p^{*}(v)=x$. There are four possibilities for $m$ : (a) $m \equiv 3(\bmod 4)$, (b) $m \equiv 2(\bmod 4)$, (c) $m \equiv 1(\bmod 4)$, and (d) $m \equiv 0(\bmod 4)$

Subcase(a): As $m \equiv 3(\bmod 4)$, we get $H^{m-2}(X / G)=H^{m-1}(X / G)=0$ and $H^{m-3}(X / G)$ $\cong H^{m-4}(X / G) \cong \mathbb{Z}_{2}$. By Lemma 4.3, $p^{*}: H^{m}(X / G) \rightarrow H^{m}(X)$ must be trivial. This gives that $H^{m}(X / G) \cong H^{m-4}(X / G)$ and $H^{m+j}(X / G)=0$ for $1 \leq j \leq 3$. Consequently, we have $H^{m+n-3}(X / G) \cong \mathbb{Z}_{2}$ and $H^{m+n-j}(X / G)=0$ for $j=1,2$ and 4 . Thus, we have

$$
H^{i}(X / G)= \begin{cases}\mathbb{Z}_{2} & \text { if } 0 \leq i \equiv 0(\bmod 4)<m, n \leq i \equiv 3(\bmod 4)<n+m \\ 0 & \text { otherwise }\end{cases}
$$

As $\cup: H^{m-3}(X / G) \rightarrow H^{m+1}(X / G)$ is trivial, we get $u^{\frac{m+1}{4}}=0$. Since $n \equiv 3(\bmod 4)$, $v^{2}=0$. Therefore, $H^{*}(X / G)$ is given by $\mathbb{Z}_{2}[u, v] /\left\langle u^{\frac{m+1}{4}}, v^{2}\right\rangle$ where $\operatorname{deg} u=4, \operatorname{deg} v=n$. This realizes possibility (i). 
Subcase(b): As $m \equiv 2(\bmod 4)$, we have $H^{m-2}(X / G)=0$. This implies that $H^{m+2}(X / G)$ $\cong H^{m-2}(X / G)$. Consequently, $H^{m+n-1}(X / G) \cong \mathbb{Z}_{2}$ which contradicts that $G$ acts freely on $X$. If $m=n+3$, then we get the same cohomology groups.

Subcase(c)\&(d): For $m \equiv 1(\bmod 4)$ or $m \equiv 0(\bmod 4)$, we get $H^{m-3}(X / G)=0$. By the exactness of the Gysin sequence, $p^{*}: H^{m}(X / G) \rightarrow H^{m}(X)$ must be nontrivial, a contradiction. We get the same result for $m=n+2$ or $m=n+1$.

Now, we consider $n=m$.

It is clear that $H^{n+j}(X / G)=0, j=2,3 . \quad$ As $H^{n}(X) \cong \mathbb{Z}_{2} \oplus \mathbb{Z}_{2}, \rho: H^{n}(X) \rightarrow$ $H^{n-3}(X / G)$ cannot be injective and so $p^{*}: H^{n}(X / G) \rightarrow H^{n}(X)$ must be nontrivial. Next, we observe that $p^{*}: H^{n}(X / G) \rightarrow H^{n}(X)$ can not be onto. Let if possible, then $H^{n}(X / G) \cong \mathbb{Z}_{2} \oplus \mathbb{Z}_{2}$. Therefore, there exist nonzero element $u, v \in H^{n}(X / G)$ such that $p^{*}(u)=x$ and $p^{*}(v)=y$. So, $p^{*}(u v)=x y \neq 0$ in $H^{2 n}(X)$. Consequently, $0 \neq u v \in$ $H^{2 n}(X / G)$ which contradicts Lemma 4.2. Therefore, $\operatorname{im}\left(p^{*}: H^{n}(X / G) \rightarrow H^{n}(X)\right) \cong \mathbb{Z}_{2}$, and hence $H^{n}(X / G) \cong \mathbb{Z}_{2}$. Let $\{v\}$ be the basis of $H^{n}(X / G)$. Then, $p^{*}(v)=x$ or $y$. As the sequence $0 \rightarrow \operatorname{ker} \rho \rightarrow H^{n}(X) \stackrel{\rho}{\rightarrow} \operatorname{im} \rho \rightarrow 0$ is split exact, we get $\operatorname{im} \rho \cong \mathbb{Z}_{2}$. This gives that $H^{n+1}(X / G)=0$. Accordingly, we get $H^{n}(X / G) \cong H^{2 n-3}(X / G)$ and $H^{2 n-j}(X / G)=0$ for $j=1,2$ and 4 . Thus, we have

$$
H^{i}(X / G)= \begin{cases}\mathbb{Z}_{2} & \text { if } 0 \leq i \equiv 0(\bmod 4)<n, n \leq i \equiv 3(\bmod 4)<2 n \\ 0 & \text { otherwise }\end{cases}
$$

As $H^{n+1}(X / G)=H^{2 n}(X / G)=0$, we have $u^{\frac{n+1}{4}}=0=v^{2}$. Therefore, $H^{*}(X / G)$ is given by $\mathbb{Z}_{2}[u, v] /\left\langle u^{\frac{n+1}{4}}, v^{2}\right\rangle$ where $\operatorname{deg} u=4, \operatorname{deg} v=n$. This realizes possibility(iii).

Case(III): As $n \equiv 0(\bmod 4), H^{n-4}(X / G) \cong \mathbb{Z}_{2}$ with basis $\left\{u^{\frac{n-4}{4}}\right\}$ and $H^{n-j}(X / G)=0$ for $1 \leq j \leq 3$. This implies that $H^{n+j}(X / G)=0$ for all $1 \leq j \leq 3$ and $p^{*}: H^{n}(X / G) \rightarrow$ $H^{n}(X)$ is surjective. Now, the sequence $0 \rightarrow \operatorname{im} \cup \rightarrow H^{n}(X / G) \stackrel{p^{*}}{\rightarrow} H^{n}(X) \rightarrow 0$ is split exact, therefore, $H^{n}(X / G) \cong \mathbb{Z}_{2} \oplus \mathbb{Z}_{2}$ with basis $\left\{u^{\frac{n}{4}}, v\right\}$ where $p^{*}(v)=x$. From this we get, $H^{i}(X / G)=0$ for $n<i \equiv j(\bmod 4)<m$ for $1 \leq j \leq 3$ and $H^{i}(X / G) \cong \mathbb{Z}_{2} \oplus \mathbb{Z}_{2}$ for $n \leq i \equiv 0(\bmod 4)<m$ with basis $\left\{v u^{\frac{i-n}{4}}, u^{\frac{i}{4}}\right\}$. As both $n$ and $m$ can not be even, there are two possibilities for $m$ : (a) $m \equiv 3(\bmod 4)$, and $m \equiv 1(\bmod 4)$

Subcase(a): As $m \equiv 3(\bmod 4)$, we get $H^{m-j}(X / G)=0$ for $j=1,2$ or 4 and $H^{m-3}(X / G)$ $\cong \mathbb{Z}_{2} \oplus \mathbb{Z}_{2}$. By Lemma 4.3, $p^{*}: H^{m}(X / G) \rightarrow H^{m}(X)$ must be trivial. By the exactness of the Gysin sequence, $H^{m+j}(X / G)=0$ for $j=0,2,3$ and $\rho: H^{m}(X) \rightarrow H^{m-3}(X / G)$ is injective. This gives that $\operatorname{ker}\left(\cup: H^{m-3}(X / G) \rightarrow H^{m+1}(X / G)\right) \cong \mathbb{Z}_{2}$ and hence $H^{m+1}(X / G) \cong \mathbb{Z}_{2}$. Consequently, $H^{n+m-j}(X / G)=0$ for $j=1,2,4$ and $H^{n+m-3}(X / G) \cong$ $H^{m+1}(X / G)$. Thus, we have

$$
H^{i}(X / G)= \begin{cases}\mathbb{Z}_{2} & \text { if } 0 \leq i \equiv 0(\bmod 4)<n, m<i \equiv 0(\bmod 4)<n+m \\ \mathbb{Z}_{2} \oplus \mathbb{Z}_{2} & \text { if } n \leq i \equiv 0(\bmod 4)<m \\ 0 & \text { otherwise }\end{cases}
$$


Note that a basis for $H^{m-3}(X / G)$ is $\left\{v u^{\frac{m-n-3}{4}}, u^{\frac{m-3}{4}}\right\}$. As $H^{m+1}(X / G) \cong \mathbb{Z}_{2}$, one basis element vanish under the map $\cup: H^{m-3}(X / G) \rightarrow H^{m+1}(X / G)$.

So, there are two cases: $\left(v u^{\frac{m-n+1}{4}}=0 \& u^{\frac{m+1}{4}} \neq 0\right)$ or $\left(v u^{\frac{m-n+1}{4}} \neq 0 \& u^{\frac{m+1}{4}}=0\right)$.

If $v u^{\frac{m-n+1}{4}}=0$ and $u^{\frac{m+1}{4}} \neq 0$ then the basis of $H^{m+1}(X / G)$ is $\left\{u^{\frac{m+1}{4}}\right\}$, and hence basis of $H^{n+m-3}(X / G)$ is $\left\{u^{\frac{n+m-3}{4}}\right\}$. It is clear that $u^{\frac{n+m+1}{4}}=0$. As $2 n \equiv 0(\bmod 4)$ and $v^{2} \in H^{2 n}(X / G)$, we have $v^{2}=\alpha v u^{\frac{n}{4}}+\beta u^{\frac{n}{2}}$ for $\alpha, \beta \in \mathbb{Z}_{2}$ and $\alpha=0$ if $2 n>m$. Therefore, the graded algebra of $X / G$ is given by

$$
H^{*}(X / G)=\frac{\mathbb{Z}_{2}[u, v]}{\left\langle u^{\frac{n+m+1}{4}}, v u^{\frac{m-n+1}{4}}, v^{2}+\alpha v u^{\frac{n}{4}}+\beta u^{\frac{n}{2}}\right\rangle}
$$

where $\operatorname{deg} u=4, \operatorname{deg} v=n, \alpha, \beta \in \mathbb{Z}_{2}$ and $\alpha=0$ if $2 n>m$. This realizes possibility (ii) of the theorem.

If $v u^{\frac{m-n+1}{4}} \neq 0$ and $u^{\frac{m+1}{4}}=0$ then the basis for $H^{m+1}(X / G)$ is $\left\{v u^{\frac{m-n+1}{4}}\right\}$ and hence the basis for $H^{n+m-3}(X / G)$ is $\left\{v u^{\frac{m-3}{4}}\right\}$. Obviously, $v u^{\frac{m+1}{4}}=0$. As above, we get $v^{2}=\alpha v u^{\frac{n}{4}}+\beta u^{\frac{n}{2}}$ for $\alpha, \beta \in \mathbb{Z}_{2}$ and $\beta=0$ if $2 n>m$. Hence, we have

$$
H^{*}(X / G)=\frac{\mathbb{Z}_{2}[u, v]}{\left\langle u^{\frac{m+1}{4}}, v^{2}+\alpha v u^{\frac{n}{4}}+\beta u^{\frac{n}{2}}\right\rangle}
$$

where $\operatorname{deg} u=4, \operatorname{deg} v=n, \alpha, \beta \in \mathbb{Z}_{2}$ and $\beta=0$ if $2 n>m$. This realizes possibility(i) of the theorem.

Subcase(b): As $m \equiv 1(\bmod 4)$, we get $H^{m-1}(X / G) \cong \mathbb{Z}_{2} \oplus \mathbb{Z}_{2}$, and $H^{m-j}(X / G)=0$ for $j=2,3$ and 4 . By the exactness of the Gysin sequence, $p^{*}: H^{m}(X / G) \rightarrow H^{m}(X)$ must be nontrivial, a contradiction. For $m=n+1$, we get the same cohomology groups.

Case(IV): When $n \equiv 2(\bmod 4)$.

As $n \equiv 2(\bmod 4)$, we get $H^{n-2}(X / G) \cong \mathbb{Z}_{2}$ with the basis $\left\{u^{\frac{n-2}{4}}\right\}$ and $H^{n-j}(X / G)=0$ for $j=1,3,4$. By the exactness of the Gysin sequence, $H^{n}(X / G) \cong H^{n}(X), H^{n+1}(X / G)=$ $H^{n+3}(X / G)=0$ and $H^{n+2}(X / G) \cong H^{n-2}(X / G)$. The Bases for $H^{n}(X / G)$ and $H^{n+2}(X / G)$ are $\{v\}$ and $\left\{u^{\frac{n+2}{4}}\right\}$, respectively, where $p^{*}(v)=x$. Further, $H^{i}(X / G)=0$ for $n<$ $i \equiv \mathrm{j}(\bmod 4)<m, j=1,3 ; H^{i}(X / G) \cong \mathbb{Z}_{2}$ for $n \leq i \equiv 2(\bmod 4)<m$ with basis $\left\{v u^{\frac{i-n}{4}}\right\}$ and $H^{i}(X / G) \cong \mathbb{Z}_{2}$ for $n<i \equiv 0(\bmod 4)<m$ with basis $\left\{u^{\frac{i}{4}}\right\}$. By Lemma 4.3, $p^{*}: H^{m}(X / G) \rightarrow H^{m}(X)$ must be trivial. Here, there are two possibilities for $m$ : (a) $m \equiv 3(\bmod 4) ;$ and $(\mathrm{b}) m \equiv 1(\bmod 4)$.

Subcase(a): As $m \equiv 3(\bmod 4)$, we get $H^{m-3}(X / G) \cong H^{m-1}(X / G) \cong \mathbb{Z}_{2}$ and $H^{m-2}(X / G)$ $=H^{m-4}(X / G)=0$. Consequently, $H^{m+3}(X / G) \cong H^{m-1}(X / G)$ and $H^{m+j}(X / G)=0$ for all $0 \leq j \leq 2$, and hence $H^{n+m-j}(X / G)=0$ for $j=1,2,4$ and $H^{n+m-3}(X / G) \cong \mathbb{Z}_{2}$.

Thus, we have

$$
H^{i}(X / G)= \begin{cases}\mathbb{Z}_{2} & \text { if } 0 \leq i \equiv 0(\bmod 4)<m, n \leq i \equiv 2(\bmod 4)<n+m \\ 0 & \text { otherwise }\end{cases}
$$


It is clear that $u^{\frac{m+1}{4}}=0$. As $2 n \equiv 0(\bmod 4)$, we get $v^{2}=\alpha u^{\frac{n}{2}}$ for $\alpha \in \mathbb{Z}_{2}$ and $\alpha=0$ if $2 n>m$. Therefore, $H^{*}(X / G)=\mathbb{Z}_{2}[u, v] /\left\langle u^{\frac{m+1}{4}}, v^{2}+\alpha u^{\frac{n}{2}}\right\rangle$ where $\operatorname{deg} u=4$ and $\operatorname{deg} v=$ $n$. This realizes possibility (i). For $m=n+1$, we get the same result.

Subcase(b): As $m \equiv 1(\bmod 4)$, we get $H^{m-j}(X / G) \cong \mathbb{Z}_{2}$ for $j=1,3$; and 0 for $j=2,4$. This gives that $H^{m}(X / G)=H^{m+1}(X / G)=0$. By the exactness of the Gysin sequence, we get $H^{m+3}(X / G) \cong H^{m-1}(X / G), H^{m+2}(X / G)=0$. Consequently, $H^{m+n-j}(X / G)=0$ for $j=1,2,4$ and $H^{m+n-3}(X / G) \cong \mathbb{Z}_{2}$. Thus, we have

$$
H^{i}(X / G)= \begin{cases}\mathbb{Z}_{2} & \text { if } 0 \leq i \equiv 0(\bmod 4)<n+m, n \leq i \equiv 2(\bmod 4)<m \\ 0 & \text { otherwise }\end{cases}
$$

It is clear that $u^{\frac{n+m+1}{4}}=v u^{\frac{m-n-3}{4}}=0$. As $2 n \equiv 0(\bmod 4)$, we get $v^{2}=\alpha u^{\frac{n}{2}}$ for $\alpha \in \mathbb{Z}_{2}$. Therefore, the graded cohomology algebra of $X / G$ is

$$
H^{*}(X / G)=\frac{\mathbb{Z}_{2}[u, v]}{\left\langle u^{\frac{n+m+1}{4}}, v u^{\frac{m-n+1}{4}}, v^{2}+\alpha u^{\frac{n}{2}}\right\rangle}
$$

where $\operatorname{deg} u=4, \operatorname{deg} v=n, \alpha \in \mathbb{Z}_{2}$ and this realizes possibility (ii). For $m=n+3$, we get the same result.

Similarly, we get the cohomological structure of the orbit spaces of free $G=\mathbb{S}^{1}$ actions on a finitistic space $X \sim_{2} \mathbb{S}^{n} \times \mathbb{S}^{m}$. For example, $G=\mathbb{S}^{1}$ acts freely on $S U(3) \sim_{2} \mathbb{S}^{3} \times \mathbb{S}^{5}$ by the component-wise multiplication but $S U(3) \not \approx \mathbb{S}^{3} \times \mathbb{S}^{5}$. Recall that the same cohomology algebra with rational coefficients is determined in [5].

Theorem 4.5. Let $X$ be a finitistic free $G$-space with $X \sim_{2} \mathbb{S}^{n} \times \mathbb{S}^{m}, 1 \leq n \leq m$, where $G=\mathbb{S}^{1}$. Then $H^{*}(X / G)$ is isomorphic to one of the following graded algebras:

(i) $\mathbb{Z}_{2}[u, v] /\left\langle u^{\frac{m+1}{2}}, v^{2}+\alpha u^{n}+\beta v u^{\frac{n}{2}}\right\rangle$, where $\operatorname{deg} u=2, \operatorname{deg} v=n, m$ is odd, $\alpha=0$ if $m \leq 2 n$ and $\beta=0$ if $n$ is odd.

(ii) $\mathbb{Z}_{2}[u, v] /\left\langle u^{\frac{n+m+1}{2}}, v u^{\frac{m-n+1}{2}}, v^{2}+\alpha u^{n}+\beta v u^{\frac{n}{2}}\right\rangle$, where $\operatorname{deg} u=2, \operatorname{deg} v=n, m-n$ is odd and $\beta=0$ if $m<2 n$ or $m$ is even.

(iii) $\mathbb{Z}_{2}[u, v] /\left\langle u^{\frac{n+1}{2}}, v^{2}\right\rangle$, where $\operatorname{deg} u=2, \operatorname{deg} v=m$ and $n$ is odd.

From the above theorems, we observe that

Remark 4.6. If $G=\mathbb{S}^{d}, d=1$ or 3 , acts freely on a finitistic space $X \sim_{2} \mathbb{S}^{n} \times \mathbb{S}^{m}, 1 \leq$ $n \leq m$, then $n \equiv d(\bmod (d+1))$ or $m \equiv d(\bmod (d+1))$ or $m-n \equiv d(\bmod (d+1))$.

Using the above cohomological calculations, we get the mod 2 cohomology index of $X$ and obtain Borsuk-Ulam type results for $G=\mathbb{S}^{d}, d=1$ or 3 , actions on a finitistic space $X \sim_{2} \mathbb{S}^{n} \times \mathbb{S}^{m}, 1 \leq n \leq m$.

Corollary 4.7. Let $X$ be a finitistic free $G$-space with $X \sim_{2} \mathbb{S}^{n} \times \mathbb{S}^{m}, 1 \leq n \leq m$, where $G=\mathbb{S}^{d}, d=1$ or 3 . Then, cohom-index ${ }_{G} X$ is

(i) $\frac{m-d}{d+1}$ if $m \equiv d(\bmod d+1)$ 
(ii) $\frac{n+m-d}{d+1}$ if $(m-n) \equiv d(\bmod \mathrm{d}+1)$

(iii) $\frac{n-d}{d+1}$ if $n \equiv d(\bmod \mathrm{d}+1)$.

Proof. By Theorem 4.4 and 4.5, $u \in H^{d+1}(X / G)$ is the Steifel-Witney class of the sphere bundle $G \hookrightarrow X \rightarrow X / G$. The result follows from the definition of cohom-index $G$.

Next, we obtain Borsuk-Ulam type results and proof follows from corollary 3.8 .

Corollary 4.8. Let $X$ be a finitistic free $G$-space with $X \sim_{2} \mathbb{S}^{n} \times \mathbb{S}^{m}, 1 \leq n \leq m$, where $G=\mathbb{S}^{d}, d=1$ or 3 . Then, there is no $G$-equivariant $f: \mathbb{S}^{(d+1) k+d} \rightarrow X$

(i) for $k \geq \frac{m+1}{d+1}$, if cohom-index ${ }_{G}=\frac{m-d}{d+1}$

(ii) for $k \geq \frac{n+m+1}{d+1}$, if cohom-index $G=\frac{n+m-d}{d+1}$

(iii) for $k \geq \frac{n+1}{d+1}$, if cohom-index $\operatorname{lox}_{G}=\frac{n-d}{d+1}$

\section{EXAMPles}

5.1. Let $G=\mathbb{S}^{d}, d=1$ or 3 , acts on $\mathbb{S}^{(d+1) n+d}$ by the standard action and trivially on $\mathbb{S}^{m}$. Then $G$ acts freely on $X=\mathbb{S}^{(d+1) n+d} \times \mathbb{S}^{m}$ by the diagonal action. Note that $X / G=\mathbb{F P}^{n} \times \mathbb{S}^{m}$, where $\mathbb{F}=\mathbb{C}$ or $\mathbb{H}$ where $d=1$ or 3 . So, $H^{*}(X / G)=$ $\mathbb{Z}_{2}[u, v] /\left\langle u^{n+1}, v^{2}\right\rangle$, where $\operatorname{deg} u=d+1$ and $\operatorname{deg} v=m$. This realizes case(i)\&(iii) of Theorem 4.4 and 4.5. So, cohom-index $\operatorname{dex}_{G}=n$. By above corollary, there is no $G$ equivariant map $f: \mathbb{S}^{(d+1) k+d} \rightarrow X$ for $k \geq n+1$. As $g: \mathbb{S}^{(d+1) n+d} \rightarrow X$ defined as $g(x)=\left(x, y_{0}\right)$ is $G$-equivariant map where $y_{0} \in \mathbb{S}^{m}$ be any point. Thus, $\operatorname{ind}_{G} X=n$.

5.2. Let $\left(a_{0}, a_{1}, \cdots, a_{n}\right)$ and $\left(b_{0}, b_{1}, \cdots, b_{m}\right)$ be sequences of integers such that $\operatorname{gcd}\left(a_{i}, b_{j}\right)=$ 1 for all $0 \leq i \leq n$ and $0 \leq j \leq m$. Then, $G=\mathbb{S}^{1}$ acts freely on $X=\mathbb{S}^{2 n+1} \times$ $\mathbb{S}^{2 m+1}$ by $\lambda .(z, w)=\left(\left(\lambda^{a_{0}} z_{0}, \lambda^{a_{1}} z_{1}, \cdots, \lambda^{a_{n}} z_{n}\right),\left(\lambda^{b_{0}} w_{0}, \lambda^{b_{1}} w_{1}, \cdots, \lambda^{b_{m}} w_{m}\right)\right)$ where $z=$ $\left(z_{0}, z_{1}, \cdots, z_{n}\right), w=\left(w_{0}, w_{1}, \cdots, w_{m}\right)$ and $z_{i}, w_{i} \in \mathbb{C}$. If $n=0$ then $X / G=L_{a_{0}}^{2 m+1}\left(b_{0}, b_{1}\right.$, $\left., \cdots, b_{m}\right)$. So, for $a_{0}$ odd, $X / G \sim_{2} \mathbb{S}^{2 m+1}$; for $p$ even but $4 \not p, X / G \sim_{2} \mathbb{R P}^{2 m+1}$; and for $4 \mid p, X / G \sim_{2} \mathbb{S}^{1} \times \mathbb{C P}^{m}$. This realizes case $(\mathrm{i})$ of theorem 4.5.

\section{REFERENCES}

1. P. E. Conner and E. E. Floyd, Fixed point free involutions and equivariant maps I, Bull. Amer. Math. Soc. 66 (1960), 416-441. MR0163310

2. P. E. Conner and E. E. Floyd, Fixed point free involutions and equivariant maps II, Trans. Amer. Math. Soc. 105 (1962), 222-228. MR0143208

3. S. Deo, Index of a finitistic space and a generalization of the topological central point theorem, J. Ramanujan Math. Soc. 28 (2013), no. 2, 223-232. MR3097427

4. T. tom Dieck, Transformation groups, De Gruyter Studies in Mathematics, 8, Walter de Gruyter \& Co., Berlin, 1987. MR0889050

5. R. M. Dotzel, T. B. Singh and S. P. Tripathi, The cohomology rings of the orbit spaces of free transformation groups of the product of two spheres, Proc. Amer. Math. Soc. 129 (2001), no. 3, 921-930. MR1712925

6. E. Fadell and S. Husseini, An ideal-valued cohomological index theory with applications to Borsuk-Ulam and Bourgin-Yang theorems, Ergodic Theory Dynam. Systems $\mathbf{8}^{*}$ (1988), Charles Conley Memorial Issue, 73-85. MR0967630

7. Y. Hara, The degree of equivariant maps, Topology Appl. 148 (2005), no. 1-3, 113-121. MR2118958

8. A. Hatcher, Algebraic topology, Cambridge University Press, Cambridge, 2002. MR1867354 
9. D. Husemoller, Fibre bundles, third edition, Graduate Texts in Mathematics, 20, Springer-Verlag, New York, 1994. MR1249482

10. J. Jaworowski, The index of free circle actions in lens spaces, Topology Appl. 123 (2002), no. 1, 125-129. MR1921653

11. J. Kaur and H. K. Singh, On the existence of free action of $\mathbb{S}^{3}$ on certain finitistic $\bmod p$ cohomology spaces, J. Indian Math. Soc. (N.S.) 82 (2015), no. 3-4, 97-106. MR3467619

12. G. R. Livesay, Fixed point free involutions on the 3-sphere, Ann. of Math. (2) 72 (1960), 603-611. MR0116343

13. B. Matschke, A parametrized version of the Borsuk-Ulam-Bourgin-Yang-Volovikov theorem, J. Topol. Anal. 6 (2014), no. 2, 263-280. MR3191652

14. J. McCleary, A user's guide to spectral sequences, second edition, Cambridge Studies in Advanced Mathematics, 58, Cambridge University Press, Cambridge, 2001. MR1793722

15. H. Ozeki and F. Uchida, Principal circle actions on a product of spheres, Osaka Math. J. 9 (1972), 379-390. MR0353348

16. P. M. Rice,Free actions of $Z_{4}$ on $S^{3}$, Duke Math. J. 36 (1969), 749-751. MR0248814

17. G. X. Ritter, Free $Z_{8}$ actions on $S^{3}$, Trans. Amer. Math. Soc. 181 (1973), 195-212. MR0321078

18. G. X. Ritter, Free actions of cyclic groups of order $2^{n}$ on $S^{1} \times S^{2}$, Proc. Amer. Math. Soc. 46 (1974), 137-140. MR0350768

19. J. H. Rubinstein,Free actions of some finite groups on $S^{3}$ I, Math. Ann. 240 (1979), no. 2, $165-175$. MR0524664

20. H. K. Singh and T. B. Singh, The cohomology of orbit spaces of certain free circle group actions, Proc. Indian Acad. Sci. Math. Sci. 122 (2012), no. 1, 79-86. MR2909586

21. S. Stolz, The level of real projective spaces, Comment. Math. Helv. 64 (1989), no. 4, 661-674. MR1023002

22. J. C. Su,Transformation groups on cohomology projective spaces, Trans. Amer. Math. Soc. 106 (1963), 305-318. MR0143839

23. R. Tanaka, On the index and co-index of sphere bundles, Kyushu J. Math. 57 (2003), no. 2, 371-382. MR2050092

24. R. Tanaka,A stability theorem for the index of sphere bundles, Bull. Belg. Math. Soc. Simon Stevin 14 (2007), no. 1, 177-182. MR2327334

25. Y. Tao, On fixed point free involutions of $S^{1} \times S^{2}$, Osaka Math. J. 14 (1962), 145-152. MR0140092

26. J. Ucci, $A$ note on fixed point free involutions and equivariant maps, Proc. Amer. Math. Soc. 31 (1972), 297-298. MR0298656

27. C. T. Yang, On theorems of Borsuk-Ulam, Kakutani-Yamabe-Yujobô and Dyson. I, Ann. of Math. (2) 60 (1954), 262-282. MR0065910

28. C. T. Yang, On theorems of Borsuk-Ulam, Kakutani-Yamabe-Yujobô and Dyson. II, Ann. of Math. (2) 62 (1955), 271-283. MR0072470

29. A. Yu. Volovikov, On the index of $G$-spaces, Sb. Math. 191 (2000), no. 9-10, 1259-1277; translated from Mat. Sb. 191 (2000), no. 9, 3-22. MR1805595

Anju Kumari, Department of Mathematics, University of Delhi, Delhi - 110007, India. Email address: anjukumari0702@gmail.com

Hemant Kumar Singh, Department of Mathematics, University of Delhi, Delhi - 110007, INDIA.

Email address: hemantksingh@maths.du.ac.in 